\title{
Dietary Patterns and Severity of Symptom with the Risk of Esophageal Squamous Cell Carcinoma and its Histological Precursor Lesions in China: A Multicenter Cross-Sectional Latent Class Analysis
}

\section{Zhaoping Zang}

Capital Medical University

Yong Liu

National Cancer Center, Chinese Academy of Medical Sciences and Peking Union Medical College

Jialin Wang

Shandong Cancer Hospital and Institute, Shandong First Medical University and Shandong Academy of Medical

Sciences

Yuqin Liu

Gansu Provincial Cancer Hospital

Shaokai Zhang

The Affiliated Cancer Hospital of Zhengzhou University

Yongzhen Zhang

Shanxi Cancer Hospital

Liwei Zhang

The Fourth Hospital of Hebei Medical University

Deli Zhao

Feicheng People's Hospital

\section{Fugang Liu}

Dongping People's Hospital

\section{Lina Chao}

Hebi People's Hospital

Xinzheng Wang

Yangcheng Cancer Hospital

\section{Chunli Zhang}

The First People's Hospital of Ningyang County

\section{Guohui Song}

Cixian Institute for Cancer Prevention and Control

\section{Zhiyi Zhang}

Gansu Wuwei Cancer Hospital

\section{Youpeng Li}

Minqin County People's Hospital

\section{Zheng Yan}

Linze County People's Hospital

\section{Yongxiu Wen}


Shandan County People's Hospital

\section{Yinyin Ge}

Gaotai County People's Hospital

Chen Niu

Capital Medical University

\section{Wei Feng}

Capital Medical University

\section{Rena Nakyeyune}

Capital Medical University

\section{Yi Shen}

Capital Medical University

\section{Yi Shao}

Capital Medical University

\section{Xiuhua Guo}

Capital Medical University

\section{Aiming Yang}

Chinese Academy of Medical Sciences

Fen Liu ( $\sim$ liufen05@ccmu.edu.cn )

Capital Medical University

\section{Guiqi Wang}

National Cancer Center, Chinese Academy of Medical Sciences and Peking Union Medical College

\section{Research Article}

Keywords: Esophageal squamous cell carcinoma, Precancerous lesions, Latent class analysis, Dietary patterns, Symptom

Posted Date: November 10th, 2021

DOI: https://doi.org/10.21203/rs.3.rs-1038856/v1

License: (c) (i) This work is licensed under a Creative Commons Attribution 4.0 International License. Read Full License 


\section{Abstract}

Background: Dietary patterns and symptoms research among Chinese with esophageal squamous cell carcinoma (ESCC) and its precursor lesions is limited, especially as it relates to multiple food consumption and multiple cooccurring symptoms. The aim of our study was to identify the dietary patterns and severity of symptom classes with the risk of esophageal squamous cell carcinoma and its histological precursor lesions, and develop a risk prediction model for different stages of esophageal disease.

Methods: We analyzed data from a multicenter cross-sectional study carried out in ESCC high incidence areas between 2017 and 2018, which included 34,707 individuals aged 40-69 years. Dietary patterns and severity of symptom classes were derived by applying a latent class analysis (LCA). A multiple logistic regression model was used to derive the odds ratio (ORs) and corresponding 95\% confidence intervals (Cls) for ESCC and the different stages of esophageal disease according to the dietary patterns and severity of symptom classes identified. We built the risk prediction model by using a nomogram.

Results: We identified five dietary patterns and three severity of symptom classes. The dietary patterns were classified as follows: "Healthy”, “Western”, “Lower consumers-combination”, "Medium consumers-combination” and "Higher consumers-combination" patterns based on the intake of foods such as red meat, vegetables and fruits. The severity of symptoms was categorized into "Asymptomatic", "Mild symptoms" and "Overt symptoms" classes based on health-related symptoms reported by the participants. Compared to the "Healthy" pattern, the other four patterns were all associated with an increased risk of esophageal disease. Similarly, the other two symptom classes present different degrees of increased risk of esophageal disease compared to the "Asymptomatic". The nomograms reflect the good predictive ability of the model.

Conclusion: Among individuals aged 40-69 years in high incidence regions of upper gastrointestinal cancer, the results supplied important epidemiological evidence and given further insights into dietary patterns and symptoms research.

\section{Background}

Esophageal cancer ranks seventh in terms of incidence and sixth in mortality overall in the world according to the report of the Global Cancer Statistics 2020. Eastern Asia shows the highest regional incidence rates for both men and women, partly because of the large burden in China [1]. In China, esophageal cancer is the sixth most common malignancy and the fourth leading cause of cancer-related deaths [2]. Esophageal adenocarcinoma (EADC) and esophageal squamous cell carcinoma (ESCC) are the two most common histologic subtypes of esophageal cancer. In China, more than $90 \%$ of esophageal cancer cases are ESCCs, whose number accounts for about half of all the ESCC cases on earth [3]. The most surveyed region of China is the North Central Taihung Mountain range. In small areas of this region, ESCC may be at or nearly the leading cause of death, with incidence rates exceeding $125 / 100,000$ per year [4]. Therefore, identification of risk factors in the early stages of the disease appears to be essential in order to decrease ESCC incidence and mortality.

Esophagitis is a precancerous disease of esophageal cancer, and it is a benign disease with a certain canceration rate. Based on WHO tumor histological classification, the esophageal precancerous lesions (EPL) can be defined as low-grade intraepithelial neoplasia (LGIN) and high-grade intraepithelial neoplasia (HGIN) [5]. With the aggravation of precancerous lesions, the rate of developing esophageal cancer increased from $24-74 \%$ [6]. Therefore, intervention at an early stage of the disease results in a significant decrease in ESCC incidence and mortality. The etiology of 
ESCC is multi-factorial and strongly population dependent. A study estimated a population-attributable risk of $89 \%$ using only cigarette smoking, alcoholic beverage consumption, and low consumption of fruits and vegetables [7]. There is also some evidence on the protective effect of fruit and vegetable and the potential harmful effect of processed/red meat consumption. The evidence on the effect of diet on ESCC risk is however still suggestive or limited [8]. Before a diagnosis of esophageal cancer is established, majority of the patients have experienced pain, dysphagia, eating difficulties, appetite loss, bloating and nausea resulting in patients' daily living and quality of life [9]. Therefore, it is more meaningful for the prevention of esophageal cancer that identifying the high risk dietary patterns and some classical symptoms in the early stage of esophageal cancer.

The analysis of dietary patterns aims to fully explore the complexity of the diet, as an alternative to the study of isolated components. These techniques depend upon the concept that food consumption can be effectively presented by reproducible patterns, in spite of individual variations, and that food eaten together may have interactive effects on the risk of cancer. Similarly, the symptoms are the same. Identifying symptom clusters and their relationship to patient characteristics may lead to a better interpretation for identifying patients with early lesions and provide greater insight into the planning of future interventions.

Latent class analysis (LCA) is a model-based cluster analysis technique that allows for identifying prevalent, mutually exclusive, eating behaviors and symptoms with additional advantages with respect to the classical approaches [10]. Unlike principal component analysis (PCA) and factor analysis (FA), it can be used to categorize individuals into mutually exclusive groups, dietary patterns or severity of symptoms and differently from cluster analysis, it grants quantification of the uncertainty of class membership, and assessment of goodness of fit [11].

Our study aims to identify dietary patterns and severity of symptom through LCA, and thus to model screening for different stages of the disease. Therefore, adding a new perspective on the association between dietary habits and symptoms and ESCC in China.

\section{Methods}

\section{Study population}

This was a multicenter cross-sectional study, depending on high incidence regions of esophageal and gastric cancer established by the cancer early diagnosis and early treatment project in China [12]. In 2017, a new screening study of upper gastrointestinal cancer in five high-risk regions of upper gastrointestinal cancer in China, including Hebei, Henan, Shandong, Shanxi, and Gansu Provinces was released. The main purpose of this project was to confirm the high-risk population of malignant upper gastrointestinal cancer and to establish a cancer risk prediction model to provide support for the prevention of upper gastrointestinal cancer.

The inclusion criteria were as follows: (1) local permanent residents in selected regions, (2) no history of endoscopic examination during the last 3 years, (3) no history of cancer, mental disorder, or any contraindication for endoscopy, (4) signed informed consent and (5) agreement to complete the entire survey and examination, including endoscopy. The participant selection process is shown in Figure 1. We recruited participants from April 2017 to December 2018. The final analysis included 34,707 residents aged 40-69 years. Among these participants, there were 81 persons with ESCC, 251 persons with HGIN, 1,413 persons with LGIN, 3,883 persons with esophagitis and 29,079 persons serving as normal esophagus controls. 
The study was approved by the Capital Medical University, Chinese Academy of Medical Sciences and Peking Union Medical College. The experimental protocol involving humans was in accordance to the guidelines of the Declaration of Helsinki.

\section{Diet and symptoms assessment}

Comprehensive questionnaire information was collected by face-to-face interviews and entered directly into a laptop based data entry system by trained investigators. The data entry process was conducted with software designed to decrease missing items and reduce logic inaccuracy. A questionnaire typically took 35-45 minutes to complete. Items of dietary intake were selected through the above questionnaire, including livestock meat, poultry meat, seafood, eggs and their products, vegetables, fruits, bean products, scallions, ginger and garlic, pickles and nuts. All the variables were categorical. Foods that are consumed more frequently, were divided into three categories namely: every day, 1-6 days per week, and less than 1 day per week. Foods that are consumed infrequently, were divided into two categories namely: at least one day per week and less than one day per week. Items of typical symptoms were also selected through the above questionnaire, including number of lost teeth, frequent bleeding of gums, dysphagia, bloating, heartburn, acid reflux, nausea, vomiting, belching and epigastric pain. The number of lost teeth were categorized into three groups namely: never dropped, 1-3 teeth and more than 4 teeth. Other variables are categorized as yes or no.

\section{Outcome assessment}

The endoscopic examinations were carried out by physicians at local hospitals. Procedures were based on clinical guidelines for cancer screening and early diagnosis and treatment in China. Lugol's iodine staining method was used to identify suspicious tissues, which were then biopsied. To confirm severity, the esophageal mucosa was ranked into 5 categories: normal esophageal mucosa, minor mucosa changes, esophagitis, esophageal squamous simple hyperplasia (ESSH) or esophageal squamous dysplasia (ESD) [13]. ESD was further classified into 3 levels including slight, moderate, and severe. According to WHO tumor histological classification, mild and moderate ESD combined fall under LGIN. Severe ESD and squamous cell carcinoma in situ are considered as HGIN. If there were any inconsistencies, a third pathologist would give advice through discussion. Doctors reported the worst biopsy diagnosis to be from participants with multiple lesions. In this study, we divided the participants into 4 groups: normal control, esophagitis, LGIN and HGIN/ESCC.

\section{Statistical methods}

We characterized the dietary patterns and symptom patterns, assumed as unobserved mutually exclusive, with different variables probability distributions, by performing LCA on the observed responses on the different items.

LCA identified latent classes of participants based on the ten dietary variables and six symptom variables. Estimation was conducted with the robust maximum-likelihood and expectation-maximization algorithms [14]. Statistical fit indexes were used to assess model fit and to decide the final number of latent classes. The model that fits the data best was selected by a combination of the following criteria: (a) the lowest Akaike information criterion (AIC), (b) the lowest Bayesian information criterion (BIC), (c) the lowest Lo-Mendell-Rubin likelihood ratio test (LMR), (d) the lowest Lo-Mendell-Rubin Adjusted LMR test (ALMR), and (e) entropy to be 0.6 or greater [15]. Next, we executed an unconditional multivariable logistic regression to identify sociodemographic and risk factors that 
predicted class membership. Before conducting the analysis, we performed a covariance diagnosis between the independent variables. We considered models to calculate the adjusted odds ratios (ORs) and 95\% confidence intervals (Cls), including age, gender, education, body mass index (BMI), smoking and drinking at the same time. We also used a nomogram to model normal controls separately from the different stages of the disease. Evaluation of the model was performed using calibration curves and decision curves. In addition, basic, descriptive statistics show categorical variables as percentages, while continuous variables are shown as mean and standard deviations.

LCA was conducted in both cases and normal controls. Analysis of only the normal control was performed to check the robustness of the previous solution. As dietary patterns and symptom severity classes identified on controls were consistent (number and characteristics of the patterns) with the ones obtained on the overall dataset, we based all our analysis on the overall dataset. To guarantee the internal reproducibility of the chosen solution the analysis was conducted separately in two randomly selected subsets of the original data several times.

Statistical analyses were performed using Mplus (version 8.1) and R (version 3.6.3) software. All tests were twosided and had a significance level of 0.05 .

\section{Results}

When fitting the LCA model, we selected the result with 5 classes for dietary patterns and 3 classes for the severity of symptom in every group according to the criteria in methods (Table 1 and 2).

\section{Latent classes of dietary patterns}

Table 3 shows the conditional distribution of food group intake for all participants, giving the latent classes for the food groups that were more relevant in discriminating and labeling the clusters. The table of the normal control and the other three groups of different stages of disease are given in the Supporting Information Table S1-S3. We identified five classes with similar probability distributions according to a previous study [10], both for all participants and for different stages of the disease. Cluster 1 labeled "Healthy pattern", showed higher probability to consume more fruiting vegetables and all other kinds of fruits, high-quality protein and lower probability to consume red meat. Subjects in Cluster 2, "Western pattern," reported higher consumption of red meat and lower consumption of vegetables and fruits. Clusters 3 to 5 were related to previous food groups, but with a difference in the amount of intake. We termed Cluster 3 "Lower consumers-combination pattern" as people in it were less likely to eat all kinds of food. Cluster 4 had slightly higher probability than cluster 3 to eat all kinds of food. We called this cluster "Medium consumers-combination pattern." Cluster 5 had the highest probability as compared to cluster 3 and 4 to eat all kinds of food, so we name it as "Higher consumers-combination pattern." The estimated cluster sizes were 28.8\% ( $n=$ 9990) for the "Healthy pattern," 9.3\% ( $n=3216)$ for the "Western pattern," 29.1\% ( $=10100)$ for the "Lower consumers-combination pattern," 28.7\% ( $n=9971)$ for the "Medium consumers-combination pattern" and $4.1 \%(n=$ 1430) for the "Higher consumers-combination pattern."

Descriptions of the clusters for selected variables are given in Table 5. The proportion of smokers to drinkers was higher in the Western pattern compared to the Healthy pattern. For the other three patterns, the proportion of smokers to drinkers gradually increased with increasing food consumption. A similar trend was shown in the other three subgroups (data not shown).

\section{Latent classes of severity of symptom}


Table 4 shows the conditional distribution of symptom group for all participants, giving the latent classes for the symptom groups more relevant in discriminating and labeling the clusters. The table of the normal control and the other three groups of the different stages of disease are given in the Supporting Information Table S4-S6. For all participants and for different stages of the disease, there were three classes with similar probability distributions. We named the first class "Asymptomatic" as subjects were reported to be relatively healthy and not showing any symptoms. Class 2 was named "Mild symptoms" as subjects in this cluster reported significant symptoms in terms of gingival bleeding. Subjects in the last class had a high percentage of symptoms reported in all areas. We named this class "Overt symptoms." Sizes of the severity of symptom classes were $71.9 \%$ for the "Asymptomatic", $26.7 \%$ for the "Mild symptoms" and $1.5 \%$ for the "Overt symptoms."

Further description of the severity of symptom classes for a selected set of variables are shown in Table 6. Differences between the clusters in demographics were not particularly significant. A similar trend was shown in the other three subgroups (data not shown).

\section{Logistic regression analysis and nomogram}

We applied an unconditional multivariable logistic regression to analyze dietary patterns as well as symptom severity for normal controls vs different stages of esophageal diseases. The results of the covariance diagnosis showed no significant covariance between the independent variables (data not shown). Table 7 and 8 shows the ORs and $95 \% \mathrm{Cl}$ for all stages of disease, by the classification in the five dietary patterns and three symptom severity classes from the composite model including the relevant confounding and risk variables. In the dietary patterns, compared to the "Healthy" pattern, the "Western", the "Lower consumers-combination", the "Medium consumerscombination" and the "Higher consumers-combination" were positively related to the risk of the progression of the disease stage for esophagitis $(\mathrm{OR}=1.42,95 \% \mathrm{Cl}$ : 1.23-1.53; OR=2.33, 95\% Cl: $2.11-2.57$; OR=1.99, 95\% Cl: $1.80-2.19$ and $\mathrm{OR}=1.59,95 \% \mathrm{Cl}$ : 1.32-1.92, respectively). Consistent results were also observed between the normal control, LGIN/HGIN and ESCC three groups. The "Western", the "Lower consumers-combination”, the "Medium consumerscombination" and the "Higher consumers-combination" patterns showed a positive association with the risk of LGIN and HGIN and ESCC compared to the "Healthy" pattern. In the symptom severity classes, compared to the "Asymptomatic" class, the "Mild symptoms" class was positively related to the risk of the progression of the disease stage for the esophagitis and LGIN groups, OR=1.87, 95\% Cl: 1.66-2.10, OR=1.25, 95\% Cl: 1.11-1.41 for the "Mild symptoms" class and $\mathrm{OR}=1.04,95 \% \mathrm{Cl}$ : 0.86-1.25 and $\mathrm{OR}=0.82,95 \% \mathrm{Cl}$ : 0.51-1.31 for the "Overt symptoms" class. However, in the HGIN\&ESCC group, the "Overt symptoms" class was positively related to the risk of HGIN\&ESCC compared to the "Asymptomatic" class (OR=1.58, 95\% Cl: 1.00-2.50), The "Mild symptoms" class did not differ significantly from the "Asymptomatic" class (OR=0.98, 95\% Cl: 0.58-1.66).

After logistic regression, we performed nomogram building and internal validation for each of the three subgroups. The model was virtually presented in the form of a nomogram (Figure 2), the C-index of the novel nomogram was $0.612,0.684$ and 0.746 , respectively for esophagitis, LGIN and HGIN\&ESCC groups, embodying the good predictive ability of the model. The calibration curves also showed good consistency in the probability between the actual observation and the nomogram prediction (Figure 3 a-c). In addition, decision curve analyses (DCA) exhibited great positive net benefits in the predictive model among almost all of the threshold probabilities at different groups, indicating the favorable potential clinical effect of the predictive model (Figure $3 \mathrm{~d}-\mathrm{f}$ ). 


\section{Discussion}

This study is the first latent class analysis based on a natural population in a high incidence area of esophageal cancer in China. Additionally, this is the largest study to use latent class analysis to describe multiple dietary patterns and symptoms experienced by high incidence areas of esophageal cancer in China. Our objective was to identify dietary patterns and severity of symptoms conceived as mutually exclusive groups of people characterized by similar food intake and symptom clusters and to compare the resulting classes in terms of different stages of disease risk.

We classified five dietary patterns. We discovered a protective effect on ESCC risk for a diet rich in vegetables and fruit consumption and associated with a low intake of red meat and pickles (Healthy pattern). Compared to this group, the "Western" pattern, distinguished by a low consumption of fruit and vegetables and high intake of red meat and pickles, was associated with an increased risk of esophagitis, LGIN, HGIN and ESCC. The"Lower consumerscombination" pattern, which showed a diet deficient in most of the foods considered, was positively related to the different stages of disease risk. The higher caloric intake of the "Higher consumers-combination" pattern, leads to an increased risk of the different stages of disease. And the "Medium consumers-combination" pattern is somewhere in between.

Our results are consistent with most of the studies evaluating the influence of diet on ESCC risk [16]. The pattern with a high intake of fruit and vegetables, named "Healthy" or "Vegetable and Fruit" was found and associated with a decrease of ESCC risk in most of these studies [17-20]. A potential adverse effect of a diet mainly based on red meat, processed food in general was also found [21, 22]. In a previous study, despite different labelling, number of components and weighting, the most commonly classified patterns were an 'unhealthy' one (often named 'Western') and a 'healthy' one in different country [23]. The other three patterns were not common in previous studies.

The "Lower consumers-combination" pattern, is usually composed of people whose dietary intake is insufficient. Previous studies have reported a significantly increased risk of esophageal cancer in malnourished populations [24]. For the "Higher consumers-combination" pattern, the increased risk of esophageal cancer may be due to the high caloric intake of food. And a case-control study conducted in an Iranian population showed that higher intake of calories and total fat significantly increased the risk of esophageal cancer [25]. The increased risk of esophageal cancer in the "Medium consumers-combination" pattern may be due to the high intake of red meat and processed foods and relatively low intake of fruits and vegetables. The reasons for similar results in the subgroup analysis can be explained in the same way.

We classified three symptom severity classes defined as mutually exclusive groups of subjects characterized by different symptoms. We found that at the stage of esophagitis and LGIN, the patient has only mild symptoms, while at the stage of HGIN and ESCC, the patient has relatively severe symptoms. If symptoms can be identified early in the disease process, the development of the disease can be reduced. In esophagitis and in the LGIN stage, compared to the "Asymptomatic" class, the "Mild symptoms" class may experience tooth loss and frequent gingival bleeding. In a study of risk factors for esophageal cancer and its precancerous lesions conducted in Henan, China, a high number of missing teeth was found to be a significant risk factor [26]. In another studies of a Chinese population, tooth loss was found to be a risk factor for esophageal squamous cell cancer [27] as well as gastric cancer [28]. The hypothesis, frequently cited in the ESCC etiology literature, is that incomplete chewing and rapid swallowing of large pieces of food might lead to irritation or damage to the esophageal epithelium and subsequently increase the risk of ESCC [29]. In the current study, the relationship between the number of teeth lost and ESCC risk supports this hypothesis. Another hypothesis is that poor oral hygiene and tooth loss mediate a bacterial load and "overgrowth" of microorganisms on teeth [30], which can transform nitrates into nitrites and then combine with amines to form 
carcinogenic nitrosamines, some of which may be gastrointestinal organ-specific carcinogens [31, 32]. Therefore, an association between tooth loss and esophageal disease seems plausible. For people with frequent gingival bleeding, the increased risk of esophageal disease may be due to poor oral health as a result of bleeding gums. And many studies have shown that poor oral health is a risk factor for the development of esophageal cancer [33-36]. In the HGIN and ESCC group, compared to the "Asymptomatic" class, the "Overt symptoms" class has an increased risk of HGIN and ESCC. In this class, each symptom has a certain percentage of occurrence, especially upper gastrointestinal symptoms such as dysphagia, nausea, vomiting, etc. This indicates that upper gastrointestinal symptoms are already present when esophageal squamous cell carcinoma occurs or is about to occur. This reinforces the importance of intervention at an early stage of the disease.

LCA can bring interesting insights into dietary patterning and symptom severity classes, allowing to identify prevalent types of eating behavior and severity of symptom in a population and to compare risk for people with different types of diet and symptom severity. Traditional methods such as PCA and FA, which have the disadvantage of not being able to produce mutually exclusive groups. Thus, when the interest is to compare subgroups of subjects, an additional step of cross-classification of the dimensions/combinations is needed. The application of a LCA to this study on different stages of esophageal disease overcomes problems inherent in the traditional methods and gives further advantages in dietary patterning and symptom severity class, such as a probability-based classification under a general parametric approach and pattern prevalence estimation.

A nomogram is a convenient tool to anticipate and quantify the chance of an individual patient progressing to a certain clinical event. Nomograms are helpful in clinical decision making and valuable in risk stratification and individualized treatment [37]. Based on the nomogram, we can calculate the individual risk score and thus estimate the individual risk of disease. The identification of individuals as patients with early-stage disease, coupled with timely interventions, leads to a decrease in the incidence of ESCC. In this study, the nomogram we created had a good predictive power. Using, age, gender, education, BMI, smoking status, drinking status, dietary patterns, and symptom severity to build the model, it exhibited great positive net benefits.

A potential limitation of our study is recall bias, which is the common issue in cross-sectional studies. The community-level recruitment approach in this report reduces but does not eliminate this source of bias. In addition, our model has only been internally validated, and the model will need to be further externally validated in the future if possible. The main strength of our study is that the study population is a natural multicenter population with a high incidence of esophageal squamous cell carcinoma in China. Moreover, we have explored both ESCC and different stages of early esophageal lesions. The detailed questionnaire was collected in a standardized data entry system by trained investigator to assure the quality of the data.

\section{Conclusions}

In conclusion, LCA gives further insights into dietary patterns and symptoms research, allowing for the definition and estimation of the prevalence of different groups of subjects characterized by different dietary choices and symptoms, and comparing those groups in relation to important health outcomes. At the same time, using the results of LCA analysis to establish a prediction model to screen high-risk individuals at the early stage of esophageal lesions, and to take intervention measures can effectively decrease the incidence and mortality of ESCC. Furthermore, cohort studies are needed to verify and expand our understanding.

\section{Abbreviations}


ESCC: esophageal squamous cell carcinoma

LCA: latent class analysis

ORs: odds ratio

Cls: confidence intervals

EADC: Esophageal adenocarcinoma

EPL: esophageal precancerous lesions

LGIN: low-grade intraepithelial neoplasia

HGIN: high-grade intraepithelial neoplasia

PCA: principal component analysis

FA: factor analysis

ESSH: esophageal squamous simple hyperplasia

ESD: esophageal squamous dysplasia

AIC: Akaike information criterion

BIC: Bayesian information criterion

BMI: body mass index

\section{Declarations}

\section{Availability of data and materials}

The datasets used and/or analysed during the current study are available from the corresponding author on reasonable request.

\section{Ethics statement}

The study was approved by the independent ethics committee of National Cancer Center/Cancer Hospital, Chinese Academy of Medical Sciences and Peking Union Medical College (approval no. 17-124/1380) and ethics committee of Capital Medical University (approval no. Z2019SY012). All participants signed informed consent. All the experiment protocol for involving humans was in accordance to guidelines of Declaration of Helsinki.

\section{Consent for publication}

Not applicable 


\section{Competing interests}

The authors declare that they have no competing interests.

\section{Funding}

This work was supported by the National Key R\&D Program of China (grant numbers 2016YFC1302804, 2016YFC1302800, 2016YFC0901400); National Natural Science Foundation of China (grant numbers 81874277, 81974493); CAMS Innovation Fund for Medical Sciences (grant numbers 2016-I2M-1-001, 2017-I2M-1-001, 2019I2M-2-004); and Sanming Project of Medicine in Shenzhen (grant number SZSM201911008).

\section{Authors' contributions}

W GQ, LF, Y AM and GXH designed this study. LY, W JL, L YQ, Z SK, Z YZ, Z LW, Z DL, L FG, C LN, W XZ, Z CL, S GH, Z $Z Y, L Y P, Y Z, W$ YX and G YY contributed to the data collection and organized the database. Z ZP, NC and FW analyzed the data. Z ZP draft the manuscript. LF, RN, Shen $Y$ and Shao $Y$ revised the manuscript. W GQ, LF and Y AM made the decision to submit for publication.

\section{Acknowledgements}

Not applicable

\section{References}

1. Sung H, Ferlay J, Siegel RL, Laversanne M, Soerjomataram I, Jemal A, Bray F: Global Cancer Statistics 2020: GLOBOCAN Estimates of Incidence and Mortality Worldwide for 36 Cancers in 185 Countries. CA Cancer J Clin 2021, 71(3):209-249.

2. Chen W, Zheng R, Baade PD, Zhang S, Zeng H, Bray F, Jemal A, Yu XQ, He J: Cancer statistics in China, 2015. $C A$ Cancer J Clin 2016, 66(2):115-132.

3. Abnet CC, Arnold M, Wei WQ: Epidemiology of Esophageal Squamous Cell Carcinoma. GASTROENTEROLOGY 2018, 154(2):360-373.

4. Blot WJ, Li JY: Some considerations in the design of a nutrition intervention trial in Linxian, People's Republic of China. Natl Cancer Inst Monogr 1985, 69:29-34.

5. Fenoglio-Preiser C, Muoz N, Carneiro F: Pathology and genetics of tumours of the digestive system. HISTOPATHOLOGY 2001, 38(6).

6. Wang GQ, Abnet CC, Shen Q, Lewin KJ, Sun XD, Roth MJ, Qiao YL, Mark SD, Dong ZW, Taylor PR et al: Histological precursors of oesophageal squamous cell carcinoma: results from a 13 year prospective follow up study in a high risk population. GUT 2005, 54(2):187-192.

7. Engel LS, Chow WH, Vaughan TL, Gammon MD, Risch HA, Stanford JL, Schoenberg JB, Mayne ST, Dubrow R, Rotterdam H et al: Population attributable risks of esophageal and gastric cancers. J Natl Cancer Inst 2003, 95(18):1404-1413. 
8. Dalmartello M, Vermunt J, Serraino D, Garavello W, Negri E, Levi F, La Vecchia C: Dietary patterns and oesophageal cancer: a multi-country latent class analysis. J Epidemiol Community Health 2020.

9. Anandavadivelan P, Lagergren P: Cachexia in patients with oesophageal cancer. NAT REV CLIN ONCOL 2016, 13(3):185-198.

10. Dalmartello M, Decarli A, Ferraroni M, Bravi F, Serraino D, Garavello W, Negri E, Vermunt J, La Vecchia C: Dietary patterns and oral and pharyngeal cancer using latent class analysis. INT J CANCER 2020, 147(3):719-727.

11. Mori M, Krumholz HM, Allore HG: Using Latent Class Analysis to Identify Hidden Clinical Phenotypes. JAMA 2020, 324(7):700-701.

12. Zou XN: Epidemic trend, screening, and early detection and treatment of cancer in Chinese population. CANCER BIOL MED 2017, 14(1):50-59.

13. Liu X, Zhang M, Ying S, Zhang C, Lin R, Zheng J, Zhang G, Tian D, Guo Y, Du C et al: Genetic Alterations in Esophageal Tissues From Squamous Dysplasia to Carcinoma. GASTROENTEROLOGY 2017, 153(1):166-177.

14. Muthen $\mathrm{B}$, Shedden $\mathrm{K}$ : Finite mixture modeling with mixture outcomes using the EM algorithm. BIOMETRICS 1999, 55(2):463-469.

15. Kim SY: Determining the Number of Latent Classes in Single- and Multi-Phase Growth Mixture Models. STRUCT EQU MODELING 2014, 21(2):263-279.

16. Liu X, Wang X, Lin S, Yuan J, Yu IT: Dietary patterns and oesophageal squamous cell carcinoma: a systematic review and meta-analysis. Br J Cancer 2014, 110(11):2785-2795.

17. Ibiebele TI, Hughes MC, Whiteman DC, Webb PM: Dietary patterns and risk of oesophageal cancers: a population-based case-control study. Br J Nutr 2012, 107(8):1207-1216.

18. Hajizadeh B, Rashidkhani B, Rad AH, Moasheri SM, Saboori H: Dietary patterns and risk of oesophageal squamous cell carcinoma: a case-control study. PUBLIC HEALTH NUTR 2010, 13(7):1107-1112.

19. Liu X, Wang X, Lin S, Lao X, Zhao J, Song Q, Su X, Tak-Sun Yl: Dietary patterns and the risk of esophageal squamous cell carcinoma: A population-based case-control study in a rural population. CLIN NUTR 2017, 36(1):260-266.

20. Bravi F, Edefonti V, Randi G, Ferraroni M, La Vecchia C, Decarli A: Dietary patterns and upper aerodigestive tract cancers: an overview and review. ANN ONCOL 2012, 23(12):3024-3039.

21. Dawsey SM, Fagundes RB, Jacobson BC, Kresty LA, Mallery SR, Paski S, van den Brandt PA: Diet and esophageal disease. Ann N Y Acad Sci 2014, 1325:127-137.

22. De Stefani E, Boffetta P, Correa P, Deneo-Pellegrini H, Ronco AL, Acosta G, Mendilaharsu M: Dietary patterns and risk of cancers of the upper aerodigestive tract: a factor analysis in Uruguay. NUTR CANCER 2013, 65(3):384389.

23. Steck SE, Murphy EA: Dietary patterns and cancer risk. NAT REV CANCER 2020, 20(2):125-138.

24. Xu J, Cao J, Wang Y, Yao X, Wang Y, He Z, Lv W, Hu J: Novel preoperative nutritional assessment tool and prognostic model for ESCC patients. J CANCER 2019, 10(17):3883-3892.

25. Jessri $M$, Rashidkhani $B$, Hajizadeh $B$, Jessri $M$, Gotay $C$ : Macronutrients, vitamins and minerals intake and risk of esophageal squamous cell carcinoma: a case-control study in Iran. NUTR J 2011, 10:137.

26. Wei WQ, Abnet CC, Lu N, Roth MJ, Wang GQ, Dye BA, Dong ZW, Taylor PR, Albert P, Qiao YL et al: Risk factors for oesophageal squamous dysplasia in adult inhabitants of a high risk region of China. GUT 2005, 54(6):759-763.

27. Chen X, Yuan Z, Lu M, Zhang Y, Jin L, Ye W: Poor oral health is associated with an increased risk of esophageal squamous cell carcinoma - a population-based case-control study in China. INT J CANCER 2017, 140(3):626- 
635.

28. Abnet CC, Qiao YL, Mark SD, Dong ZW, Taylor PR, Dawsey SM: Prospective study of tooth loss and incident esophageal and gastric cancers in China. Cancer Causes Control 2001, 12(9):847-854.

29. Abnet CC, Qiao YL, Dawsey SM, Dong ZW, Taylor PR, Mark SD: Tooth loss is associated with increased risk of total death and death from upper gastrointestinal cancer, heart disease, and stroke in a Chinese populationbased cohort. INT J EPIDEMIOL 2005, 34(2):467-474.

30. Peterson LA: Formation, repair, and genotoxic properties of bulky DNA adducts formed from tobacco-specific nitrosamines. J Nucleic Acids 2010, 2010.

31. Zetterquist W, Marteus H, Kalm-Stephens P, Nas E, Nordvall L, Johannesson M, Alving K: Oral bacteria-the missing link to ambiguous findings of exhaled nitrogen oxides in cystic fibrosis. Respir Med 2009, 103(2):187193.

32. Sanchez GA, Miozza VA, Delgado A, Busch L: Total salivary nitrates and nitrites in oral health and periodontal disease. Nitric Oxide 2014, 36:31-35.

33. Ekheden I, Yang X, Chen H, Chen X, Yuan Z, Jin L, Lu M, Ye W: Associations Between Gastric Atrophy and Its Interaction With Poor Oral Health and the Risk for Esophageal Squamous Cell Carcinoma in a High-Risk Region of China: A Population-Based Case-Control Study. AM J EPIDEMIOL 2020, 189(9):931-941.

34. Mmbaga BT, Mwasamwaja A, Mushi G, Mremi A, Nyakunga G, Kiwelu I, Swai R, Kiwelu G, Mustapha S, Mghase E et al: Missing and decayed teeth, oral hygiene and dental staining in relation to esophageal cancer risk: ESCCAPE case-control study in Kilimanjaro, Tanzania. INT J CANCER 2020.

35. Dar NA, Islami F, Bhat GA, Shah IA, Makhdoomi MA, Iqbal B, Rafiq R, Lone MM, Abnet CC, Boffetta P: Poor oral hygiene and risk of esophageal squamous cell carcinoma in Kashmir. Br J Cancer 2013, 109(5):1367-1372.

36. Abnet CC, Kamangar F, Islami F, Nasrollahzadeh D, Brennan P, Aghcheli K, Merat S, Pourshams A, Marjani HA, Ebadati A et al: Tooth loss and lack of regular oral hygiene are associated with higher risk of esophageal squamous cell carcinoma. Cancer Epidemiol Biomarkers Prev 2008, 17(11):3062-3068.

37. Tang X, Zhou X, Li Y, Tian X, Wang Y, Huang M, Ren L, Zhou L, Ding Z, Zhu J et al: A Novel Nomogram and Risk Classification System Predicting the Cancer-Specific Survival of Patients with Initially Diagnosed Metastatic Esophageal Cancer: A SEER-Based Study. ANN SURG ONCOL 2019, 26(2):321-328.

\section{Tables}

Table 1

Model fit information for latent class analysis of dietary patterns 


\begin{tabular}{|c|c|c|c|c|c|c|}
\hline \multirow[t]{2}{*}{ Subtype } & Model & AIC & $\mathrm{BIC}$ & Entropy & LMR & ALMR \\
\hline & & & & & P-value & P-value \\
\hline \multirow[t]{7}{*}{ Overall } & 1-Class & 403812.534 & 403930.899 & & & \\
\hline & 2-Class & 386659.699 & 386904.886 & 0.597 & 0.0000 & 0.0000 \\
\hline & 3-Class & 380960.196 & 381332.203 & 0.628 & 0.0000 & 0.0000 \\
\hline & 4-Class & 377988.054 & 378486.881 & 0.672 & 0.0000 & 0.0000 \\
\hline & 5-Class & 376775.273 & 377400.920 & 0.648 & 0.0000 & 0.0000 \\
\hline & 6-Class & 376013.990 & 376766.458 & 0.630 & 0.0499 & 0.0506 \\
\hline & 7-Class & 375382.845 & 376262.134 & 0.616 & 1.0000 & 1.0000 \\
\hline \multirow[t]{6}{*}{ Esophagitis } & 1-Class & 383546.414 & 383664.057 & & & \\
\hline & 2-Class & 367382.696 & 367626.386 & 0.596 & 0.0000 & 0.0000 \\
\hline & 3-Class & 361925.857 & 362295.594 & 0.629 & 0.0000 & 0.0000 \\
\hline & 4-Class & 359187.987 & 359683.771 & 0.671 & 0.0000 & 0.0000 \\
\hline & 5-Class & 358056.094 & 358677.925 & 0.648 & 0.0000 & 0.0000 \\
\hline & 6-Class & 357318.205 & 358066.082 & 0.632 & 1.0000 & 1.0000 \\
\hline \multirow[t]{7}{*}{ LGIN } & 1-Class & 354511.583 & 354628.136 & & & \\
\hline & 2-Class & 339894.092 & 340135.524 & 0.590 & 0.0000 & 0.0000 \\
\hline & 3-Class & 335082.275 & 335448.584 & 0.619 & 0.0000 & 0.0000 \\
\hline & 4-Class & 332591.286 & 333082.474 & 0.662 & 0.0000 & 0.0000 \\
\hline & 5-Class & 331522.734 & 332138.800 & 0.638 & 0.0000 & 0.0000 \\
\hline & 6-Class & 330813.727 & 331554.671 & 0.623 & 0.0666 & 0.0674 \\
\hline & 7-Class & 330280.873 & 331146.696 & 0.605 & 0.7604 & 0.7604 \\
\hline \multirow[t]{7}{*}{ HGIN and ESCC } & 1-Class & 341936.596 & 342052.644 & & & \\
\hline & 2-Class & 327933.965 & 328174.349 & 0.588 & 0.0000 & 0.0000 \\
\hline & 3-Class & 323291.949 & 323656.671 & 0.620 & 0.0000 & 0.0000 \\
\hline & 4-Class & 321000.163 & 321489.221 & 0.659 & 0.0000 & 0.0000 \\
\hline & 5-Class & 319970.157 & 320583.552 & 0.638 & 0.0000 & 0.0000 \\
\hline & 6-Class & 319270.147 & 320007.879 & 0.626 & 0.0687 & 0.0696 \\
\hline & 7-Class & 318754.107 & 319616.176 & 0.605 & 0.5446 & 0.5464 \\
\hline
\end{tabular}

Note: LGIN: low-grade intraepithelial neoplasia; HGIN: high-grade intraepithelial neoplasia; ESCC: esophageal squamous cell carcinoma; AIC: akaike information criterion; BIC: bayesian information criterion; LMR: Lo-MendellRubin likelihood ratio test; ALMR: Lo-Mendell-Rubin Adjusted LMR test 
Model fit information for latent class analysis of symptom classes

\begin{tabular}{|c|c|c|c|c|c|c|}
\hline \multirow[t]{2}{*}{ Subtype } & Model & AIC & $\mathrm{BIC}$ & Entropy & LMR & ALMR \\
\hline & & & & & P-value & P-value \\
\hline \multirow[t]{4}{*}{ Overall } & 1-Class & 136196.132 & 136255.315 & & & \\
\hline & 2-Class & 134911.554 & 135038.375 & 0.898 & 0.0000 & 0.0000 \\
\hline & 3-Class & 134613.394 & 134807.852 & 0.935 & 0.0000 & 0.0000 \\
\hline & 4-Class & 134595.780 & 134857.876 & 0.623 & 0.1470 & 0.1508 \\
\hline \multirow[t]{4}{*}{ Esophagitis } & 1-Class & 129391.888 & 129450.710 & & & \\
\hline & 2-Class & 128168.972 & 128295.018 & 0.896 & 0.0000 & 0.0000 \\
\hline & 3-Class & 127893.905 & 128087.176 & 0.821 & 0.0000 & 0.0000 \\
\hline & 4-Class & 127881.486 & 128141.983 & 0.620 & 0.5484 & 0.5522 \\
\hline \multirow[t]{4}{*}{ LGIN } & 1-Class & 120040.246 & 120098.523 & & & \\
\hline & 2-Class & 118822.702 & 118947.580 & 0.890 & 0.0000 & 0.0000 \\
\hline & 3-Class & 118587.299 & 118778.779 & 0.932 & 0.0000 & 0.0000 \\
\hline & 4-Class & 118569.312 & 118827.393 & 0.648 & 0.0750 & 0.0778 \\
\hline \multirow[t]{4}{*}{ HGIN and ESCC } & 1-Class & 115838.134 & 115896.158 & & & \\
\hline & 2-Class & 114651.925 & 114776.262 & 0.889 & 0.0000 & 0.0000 \\
\hline & 3-Class & 114426.629 & 114617.279 & 0.850 & 0.0000 & 0.0000 \\
\hline & 4-Class & 114414.785 & 114671.748 & 0.688 & 0.1674 & 0.1721 \\
\hline
\end{tabular}

Note: LGIN: low-grade intraepithelial neoplasia; HGIN: high-grade intraepithelial neoplasia; ESCC: esophageal squamous cell carcinoma; AIC: akaike information criterion; BIC: bayesian information criterion; LMR: Lo-MendellRubin likelihood ratio test; ALMR: Lo-Mendell-Rubin Adjusted LMR test

Table 3

Probabilities of consumption for selected food items by dietary patterns derived from LCA for all participants 


\begin{tabular}{|c|c|c|c|c|c|c|}
\hline & & Healthy & Western & $\begin{array}{l}\text { Lower } \\
\text { consumers- } \\
\text { combination }\end{array}$ & $\begin{array}{l}\text { Medium } \\
\text { consumers- } \\
\text { combination }\end{array}$ & $\begin{array}{l}\text { Higher } \\
\text { consumers- } \\
\text { combination }\end{array}$ \\
\hline \multirow[t]{3}{*}{ Livestock meat } & everyday & $77.1 \%$ & $84.7 \%$ & $14.8 \%$ & $9.8 \%$ & $54.0 \%$ \\
\hline & $\begin{array}{l}\text { 1- } \\
\text { 6days/week }\end{array}$ & $12.5 \%$ & $12.6 \%$ & $22.3 \%$ & $72.2 \%$ & $43.3 \%$ \\
\hline & $<1$ day/week & $10.4 \%$ & $2.7 \%$ & $62.9 \%$ & $18.0 \%$ & $2.7 \%$ \\
\hline \multirow[t]{2}{*}{ Poultry meat } & $\geq 1$ day/week & $7.0 \%$ & $7.4 \%$ & $1.5 \%$ & $3.3 \%$ & $89.1 \%$ \\
\hline & $<1$ day/week & $93.0 \%$ & $92.6 \%$ & $98.5 \%$ & $96.7 \%$ & $10.9 \%$ \\
\hline \multirow[t]{2}{*}{ Seafood } & $\geq 1$ day/week & $1.5 \%$ & $9.1 \%$ & $1.5 \%$ & $0.3 \%$ & $64.8 \%$ \\
\hline & <1day/week & $98.5 \%$ & $90.9 \%$ & $98.5 \%$ & $99.7 \%$ & $35.2 \%$ \\
\hline \multirow[t]{3}{*}{ Eggs and their products } & everyday & $61.3 \%$ & $73.1 \%$ & $22.0 \%$ & $31.9 \%$ & $50.1 \%$ \\
\hline & $\begin{array}{l}\text { 1- } \\
\text { 6days/week }\end{array}$ & $15.2 \%$ & $16.4 \%$ & $24.1 \%$ & $64.5 \%$ & $38.4 \%$ \\
\hline & $<1$ day/week & $23.5 \%$ & $10.5 \%$ & $53.9 \%$ & $3.6 \%$ & $11.5 \%$ \\
\hline \multirow[t]{3}{*}{ Vegetables } & everyday & $99.8 \%$ & $0.0 \%$ & $2.5 \%$ & $2.7 \%$ & $97.2 \%$ \\
\hline & $\begin{array}{l}\text { 1- } \\
\text { 6days/week }\end{array}$ & $0.1 \%$ & $0.6 \%$ & $2.6 \%$ & $97.3 \%$ & $2.3 \%$ \\
\hline & $<1$ day/week & $0.1 \%$ & $99.4 \% \%$ & $94.9 \%$ & $0.0 \%$ & $0.5 \%$ \\
\hline \multirow[t]{3}{*}{ Fruits } & everyday & $41.4 \%$ & $18.6 \%$ & $3.0 \%$ & $2.8 \%$ & $31.8 \%$ \\
\hline & $\begin{array}{l}\text { 1- } \\
\text { 6days/week }\end{array}$ & $21.0 \%$ & $41.4 \%$ & $11.9 \%$ & $59.5 \%$ & $54.8 \%$ \\
\hline & $<1$ day/week & $37.6 \%$ & $40.0 \%$ & $85.1 \%$ & $37.7 \%$ & $13.4 \%$ \\
\hline \multirow[t]{2}{*}{ Bean products } & $\geq 1$ day/week & $38.0 \%$ & $46.3 \%$ & $8.6 \%$ & $45.3 \%$ & $65.7 \%$ \\
\hline & <1day/week & $62.0 \%$ & $53.7 \%$ & $91.4 \%$ & $54.7 \%$ & $34.3 \%$ \\
\hline \multirow[t]{2}{*}{ Scallion, ginger and garlic } & $\geq 1$ day/week & $93.7 \%$ & $72.7 \%$ & $36.6 \%$ & $59.9 \%$ & $84.7 \%$ \\
\hline & $<1$ day/week & $6.3 \%$ & $27.3 \%$ & $63.4 \%$ & $40.1 \%$ & $15.3 \%$ \\
\hline \multirow[t]{2}{*}{ Pickles } & $\geq 1$ day/week & $4.8 \%$ & $68.8 \%$ & $3.1 \%$ & $11.7 \%$ & $17.9 \%$ \\
\hline & <1day/week & $95.2 \%$ & $31.2 \%$ & $96.9 \%$ & $88.3 \%$ & $82.1 \%$ \\
\hline \multirow[t]{2}{*}{ Nut fruits } & $\geq 1$ day/week & $16.9 \%$ & $33.8 \%$ & $2.1 \%$ & $5.0 \%$ & $24.2 \%$ \\
\hline & <1day/week & $83.1 \%$ & $66.2 \%$ & $97.9 \%$ & $95.0 \%$ & $75.8 \%$ \\
\hline Cluster's size & & $28.8 \%$ & $9.3 \%$ & $29.1 \%$ & $28.7 \%$ & $4.1 \%$ \\
\hline
\end{tabular}

Table 4 
Probabilities of consumption for selected symptom items by severity of symptom derived from LCA for all participants

\begin{tabular}{|c|c|c|c|c|}
\hline & & Asymptomatic & $\begin{array}{l}\text { Mild } \\
\text { symptoms }\end{array}$ & Overt symptoms \\
\hline \multirow[t]{3}{*}{ Number of lost teeth } & 0 teeth & $48.4 \%$ & $39.5 \%$ & $32.8 \%$ \\
\hline & 1-3 teeth & $29.5 \%$ & $39.3 \%$ & $31.6 \%$ \\
\hline & $\begin{array}{l}\text { more than } 4 \\
\text { teeth }\end{array}$ & $22.1 \%$ & $21.1 \%$ & $35.6 \%$ \\
\hline \multirow[t]{2}{*}{ Whether gingival bleeding } & No & $100.0 \%$ & $0.0 \%$ & $64.9 \%$ \\
\hline & Yes & $0.0 \%$ & $100.0 \%$ & $35.1 \%$ \\
\hline \multirow[t]{2}{*}{ Whether dysphagia } & Yes & $0.2 \%$ & $0.1 \%$ & $7.0 \%$ \\
\hline & No & $99.8 \%$ & $99.9 \%$ & $93.0 \%$ \\
\hline \multirow{2}{*}{$\begin{array}{l}\text { Whether bloating, heartburn, acid } \\
\text { reflux }\end{array}$} & Yes & $1.6 \%$ & $1.4 \%$ & $47.6 \%$ \\
\hline & No & $98.4 \%$ & $98.6 \%$ & $52.4 \%$ \\
\hline \multirow{2}{*}{$\begin{array}{l}\text { Whether nausea, vomiting and } \\
\text { belching }\end{array}$} & Yes & $0.6 \%$ & $1.0 \%$ & $36.6 \%$ \\
\hline & No & $99.4 \%$ & $99.0 \%$ & $63.4 \%$ \\
\hline \multirow[t]{2}{*}{ Whether epigastric pain } & Yes & $0.8 \%$ & $0.8 \%$ & $20.1 \%$ \\
\hline & No & $99.2 \%$ & $99.2 \%$ & $79.9 \%$ \\
\hline Cluster's size & & $71.9 \%$ & $26.7 \%$ & $1.5 \%$ \\
\hline
\end{tabular}

Table 5

Dietary patterns' characteristics according to selected sociodemographic variables for all participants 


\begin{tabular}{|c|c|c|c|c|c|c|}
\hline & & Healthy & Western & $\begin{array}{l}\text { Lower } \\
\text { consumers- } \\
\text { combination }\end{array}$ & $\begin{array}{l}\text { Medium } \\
\text { consumers- } \\
\text { combination }\end{array}$ & $\begin{array}{l}\text { Higher } \\
\text { consumers- } \\
\text { combination }\end{array}$ \\
\hline & Normal control & $89.1 \%$ & $84.0 \%$ & $79.7 \%$ & $82.4 \%$ & $84.1 \%$ \\
\hline & Esophagitis & $6.9 \%$ & $9.7 \%$ & $14.7 \%$ & $12.6 \%$ & $10.4 \%$ \\
\hline & LGIN & $3.3 \%$ & $5.1 \%$ & $4.5 \%$ & $4.0 \%$ & $4.5 \%$ \\
\hline & $\mathrm{HGIN}$ and ESCC & $0.7 \%$ & $1.2 \%$ & $1.1 \%$ & $1.0 \%$ & $1.0 \%$ \\
\hline \multirow[t]{3}{*}{ Age } & $<51$ & $33.4 \%$ & $28.0 \%$ & $27.4 \%$ & $33.1 \%$ & $33.8 \%$ \\
\hline & $51-60$ & $44.3 \%$ & $45.3 \%$ & $42.6 \%$ & $41.6 \%$ & $41.3 \%$ \\
\hline & $>60$ & $22.3 \%$ & $26.7 \%$ & $30.0 \%$ & $25.3 \%$ & $24.9 \%$ \\
\hline \multirow[t]{2}{*}{ Gender } & Male & $41.3 \%$ & $46.4 \%$ & $40.0 \%$ & $38.9 \%$ & $46.0 \%$ \\
\hline & Female & $58.7 \%$ & $53.6 \%$ & $60.0 \%$ & $61.1 \%$ & $54.0 \%$ \\
\hline \multirow[t]{4}{*}{ Education } & $\begin{array}{l}\text { Never been to } \\
\text { school }\end{array}$ & $14.9 \%$ & $13.3 \%$ & $16.1 \%$ & $14.5 \%$ & $12.0 \%$ \\
\hline & Primary school & $34.3 \%$ & $33.0 \%$ & $39.6 \%$ & $35.4 \%$ & $30.8 \%$ \\
\hline & Middle school & $39.8 \%$ & $43.9 \%$ & $35.6 \%$ & $38.7 \%$ & $42.2 \%$ \\
\hline & $\begin{array}{l}\text { High school and } \\
\text { above }\end{array}$ & $11.0 \%$ & $9.8 \%$ & $8.7 \%$ & $11.4 \%$ & $15.0 \%$ \\
\hline \multirow[t]{4}{*}{ BMI } & $<18.5$ & $1.8 \%$ & $2.0 \%$ & $1.8 \%$ & $1.9 \%$ & $2.0 \%$ \\
\hline & $18.6-23.9$ & $46.6 \%$ & $44.1 \%$ & $48.9 \%$ & $44.7 \%$ & $48.3 \%$ \\
\hline & $24.0-26.9$ & $39.7 \%$ & $40.1 \%$ & $39.4 \%$ & $40.9 \%$ & $39.7 \%$ \\
\hline & $>27.0$ & $11.9 \%$ & $13.8 \%$ & $9.9 \%$ & $12.5 \%$ & $10.1 \%$ \\
\hline \multirow{3}{*}{$\begin{array}{l}\text { Smoking } \\
\text { status }\end{array}$} & Never smoked & $74.9 \%$ & $64.5 \%$ & $77.5 \%$ & $77.5 \%$ & $73.2 \%$ \\
\hline & Current smoking & $20.6 \%$ & $27.1 \%$ & $19.9 \%$ & $18.4 \%$ & $22.0 \%$ \\
\hline & Previous smoking & $4.5 \%$ & $8.4 \%$ & $2.7 \%$ & $4.1 \%$ & $4.8 \%$ \\
\hline \multirow[t]{3}{*}{$\begin{array}{l}\text { Drinking } \\
\text { status }\end{array}$} & $\begin{array}{l}\text { No alcohol } \\
\text { consumption }\end{array}$ & $62.0 \%$ & $57.6 \%$ & $75.6 \%$ & $63.9 \%$ & $64.3 \%$ \\
\hline & $<1$ time/week & $28.8 \%$ & $20.1 \%$ & $19.5 \%$ & $29.1 \%$ & $20.0 \%$ \\
\hline & $\geq 1$ time/week & $9.2 \%$ & $22.3 \%$ & $4.95 \%$ & $7.0 \%$ & $15.7 \%$ \\
\hline
\end{tabular}

Note: LGIN: low-grade intraepithelial neoplasia; HGIN: high-grade intraepithelial neoplasia; ESCC: esophageal squamous cell carcinoma; BMl: body mass index.

Table 6

Severity of symptoms' characteristics according to selected sociodemographic variables for all participants 


\begin{tabular}{|c|c|c|c|c|}
\hline & & Asymptomatic & Mild symptoms & Overt symptoms \\
\hline & Normal control & $84.0 \%$ & $82.8 \%$ & $89.1 \%$ \\
\hline & Esophagitis & $11.0 \%$ & $11.9 \%$ & $5.9 \%$ \\
\hline & LGIN & $4.0 \%$ & $4.5 \%$ & $3.8 \%$ \\
\hline & HGIN and ESCC & $1.0 \%$ & $0.8 \%$ & $1.2 \%$ \\
\hline \multirow[t]{3}{*}{ Age } & $<51$ & $30.3 \%$ & $33.5 \%$ & $25.9 \%$ \\
\hline & $51-60$ & $43.1 \%$ & $43.0 \%$ & $40.5 \%$ \\
\hline & $>60$ & $26.6 \%$ & $23.5 \%$ & $33.6 \%$ \\
\hline \multirow[t]{2}{*}{ Gender } & Male & $41.9 \%$ & $38.5 \%$ & $36.6 \%$ \\
\hline & Female & $58.1 \%$ & $61.5 \%$ & $63.4 \%$ \\
\hline \multirow[t]{4}{*}{ Education } & Never been to school & $15.0 \%$ & $13.8 \%$ & $25.5 \%$ \\
\hline & Primary school & $35.0 \%$ & $38.4 \%$ & $33.6 \%$ \\
\hline & Middle school & $39.0 \%$ & $38.4 \%$ & $29.4 \%$ \\
\hline & High school and above & $11.0 \%$ & $9.4 \%$ & $11.5 \%$ \\
\hline \multirow[t]{4}{*}{ BMI } & $<18.5$ & $1.8 \%$ & $1.8 \%$ & $4.0 \%$ \\
\hline & $18.6-23.9$ & $46.0 \%$ & $47.8 \%$ & $51.0 \%$ \\
\hline & $24.0-26.9$ & $40.1 \%$ & $39.9 \%$ & $36.4 \%$ \\
\hline & $>27.0$ & $12.1 \%$ & $10.5 \%$ & $8.7 \%$ \\
\hline \multirow[t]{3}{*}{ Smoking status } & Never smoked & $74.2 \%$ & $78.5 \%$ & $74.9 \%$ \\
\hline & Current smoking & $21.5 \%$ & $17.6 \%$ & $19.0 \%$ \\
\hline & Previous smoking & $4.3 \%$ & $3.9 \%$ & $6.1 \%$ \\
\hline \multirow[t]{3}{*}{ Drinking status } & No alcohol consumption & $65.5 \%$ & $67.9 \%$ & $70.6 \%$ \\
\hline & $<1$ time/week & $24.6 \%$ & $26.0 \%$ & $25.7 \%$ \\
\hline & $\geq 1$ time/week & $9.9 \%$ & $6.1 \%$ & $3.8 \%$ \\
\hline
\end{tabular}

Note: LGIN: low-grade intraepithelial neoplasia; HGIN: high-grade intraepithelial neoplasia; ESCC: esophageal squamous cell carcinoma; BMl: body mass index.

Table 7

Odds ratios (OR) and the corresponding 95\% confidence intervals (Cls) of esophageal cancer for each cluster of dietary patterns adjusting for known confounders: per disease stage 


\begin{tabular}{|c|c|c|c|c|}
\hline & & \multicolumn{3}{|c|}{ Adjusted OR(95\%Cl) } \\
\hline & & \multicolumn{3}{|l|}{ Disease stage } \\
\hline & & Esophagitis & LGIN & HGIN and ESCC \\
\hline & Case/Control & $3883 / 29079$ & $1413 / 29079$ & $332 / 29079$ \\
\hline \multirow[t]{5}{*}{ Dietary Patterns } & Healthy & 1 & 1 & 1 \\
\hline & Western & $1.42(1.23-1.53)$ & $1.54(1.27-1.87)$ & $1.69(1.14-2.52)$ \\
\hline & Lower consumers-combination & $2.33(2.11-2.57)$ & $1.39(1.20-1.61)$ & $1.72(1.26-2.36)$ \\
\hline & Medium consumers-combination & $1.99(1.80-2.19)$ & $1.30(1.12-1.51)$ & $1.64(1.19-2.24)$ \\
\hline & Higher consumers-combination & $1.59(1.32-1.92)$ & $1.44(1.09-1.90)$ & $1.62(0.92-2.86)$ \\
\hline
\end{tabular}

Note: LGIN: low-grade intraepithelial neoplasia; HGIN: high-grade intraepithelial neoplasia; ESCC: esophageal squamous cell carcinoma.

Table 8

Odds ratios (OR) and the corresponding $95 \%$ confidence intervals (Cls) of esophageal cancer for each cluster of symptom clusters adjusting for known confounders: per disease stage

\begin{tabular}{|lllll|}
\hline & \multicolumn{4}{l|}{ Adjusted OR(95\% Cl) } \\
\hline & & Disease stage & \\
\hline \multirow{2}{*}{ Severity of symptom } & Esophagitis & LGIN & HGIN and ESCC \\
\cline { 2 - 5 } & Case/Control & $3883 / 29079$ & $1413 / 29079$ & $332 / 29079$ \\
\cline { 2 - 5 } & Mild symptomatic & 1 & 1 & 1 \\
\cline { 2 - 5 } & Overt symptoms & $1.04(0.86-1.25)$ & $0.82(0.51-1.31)$ & $1.58(1.00-2.50)$ \\
\cline { 2 - 5 }
\end{tabular}

Note: LGIN: low-grade intraepithelial neoplasia; HGIN: high-grade intraepithelial neoplasia; ESCC: esophageal squamous cell carcinoma.

\section{Figures}




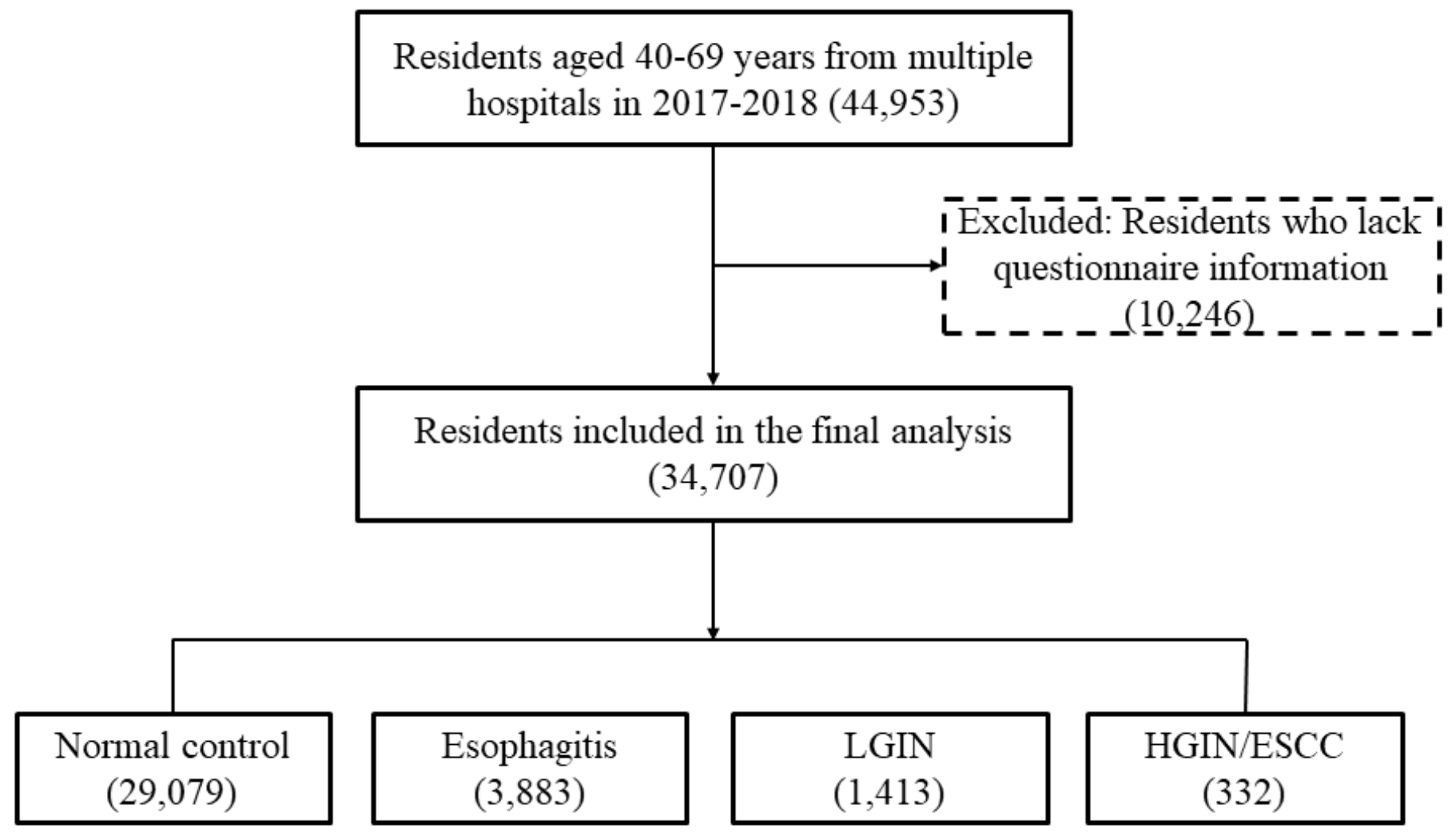

Figure 1

Flow chart of participant selection. Note: LGIN = low-grade intraepithelial neoplasia; HGIN = high-grade intraepithelial neoplasia; ESCC = esophageal squamous cell carcinoma. 
A

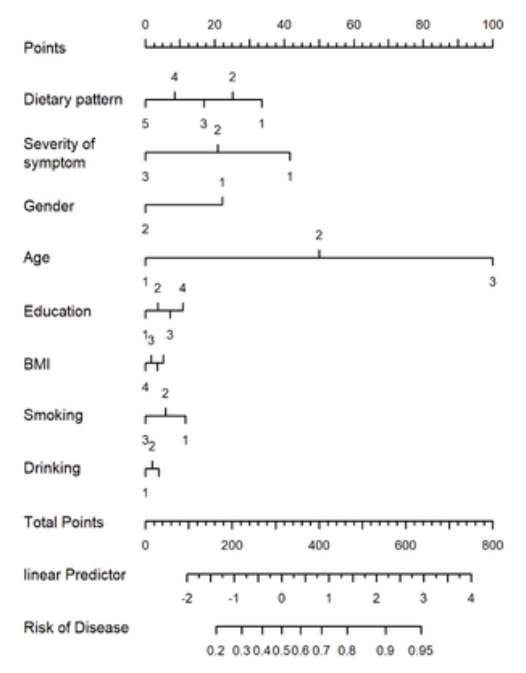

B

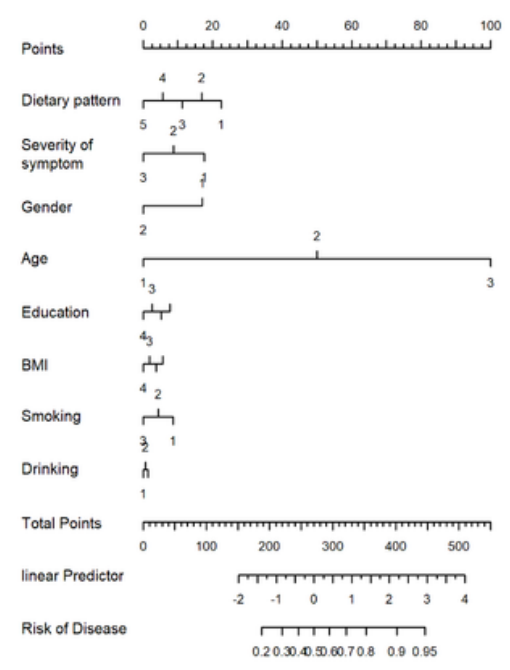

$\mathrm{C}$

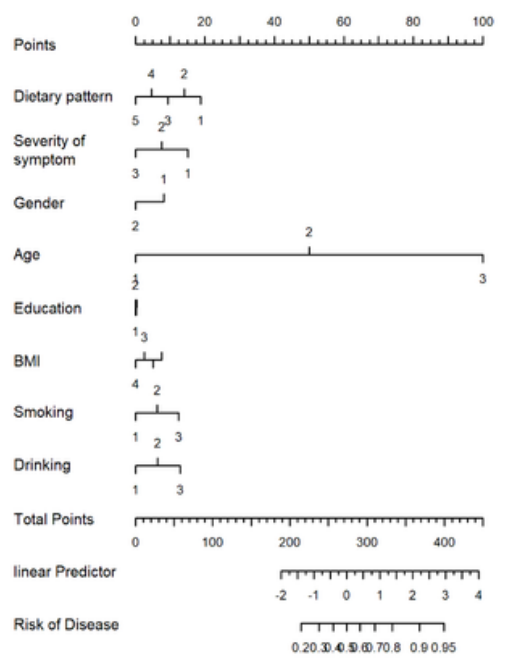

\section{Figure 2}

A: Nomogram predicting the risk for the normal control group vs esophagitis; B: Nomogram predicting the risk for the normal control group vs LGIN; C: Nomogram predicting the risk for the normal control group vs HGIN and ESCC. Note: LGIN: Iow-grade intraepithelial neoplasia; HGIN: high-grade intraepithelial neoplasia; ESCC: esophageal squamous cell carcinoma. 
A

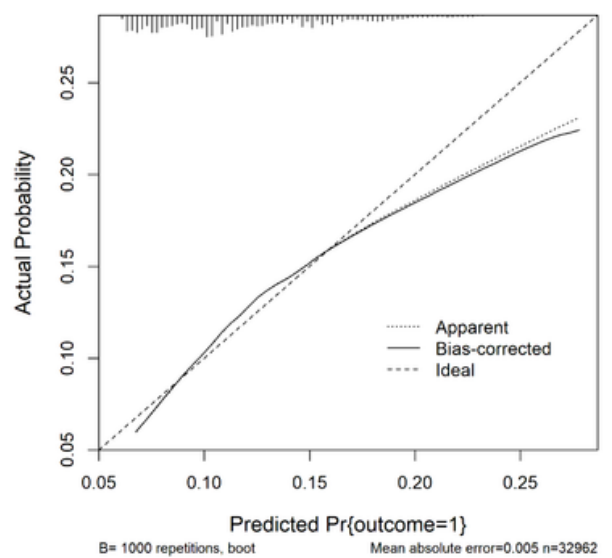

$\mathrm{D}$

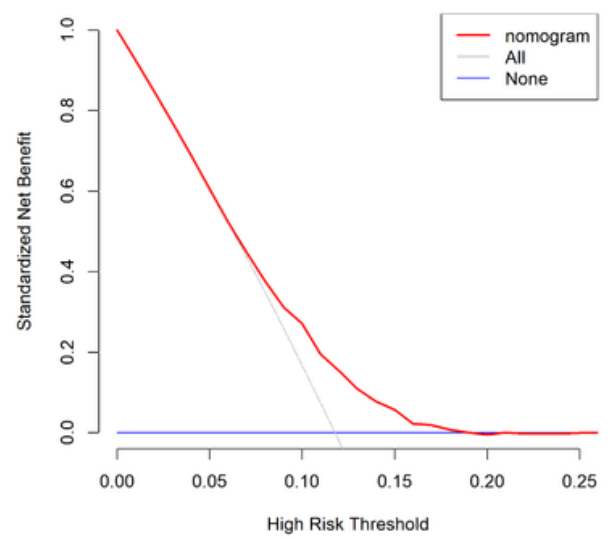

B

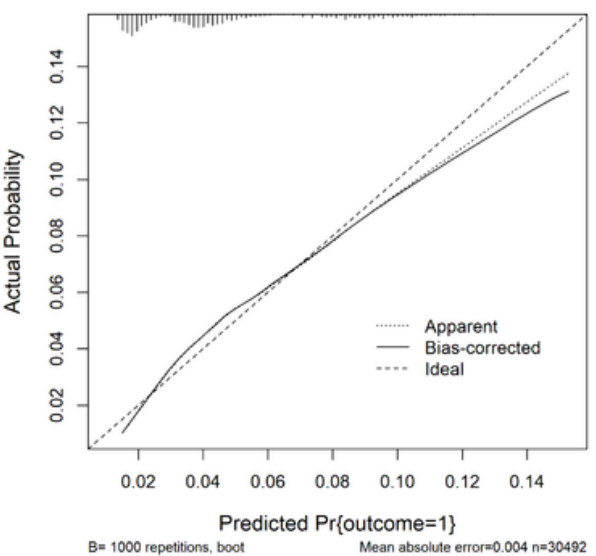

$\mathrm{E}$

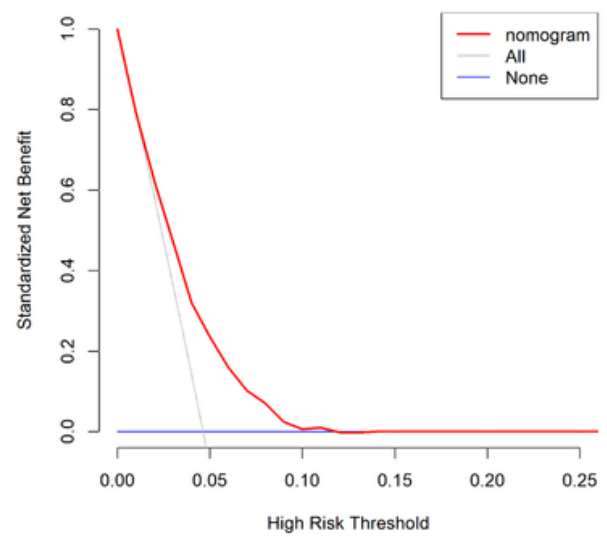

$\mathrm{C}$

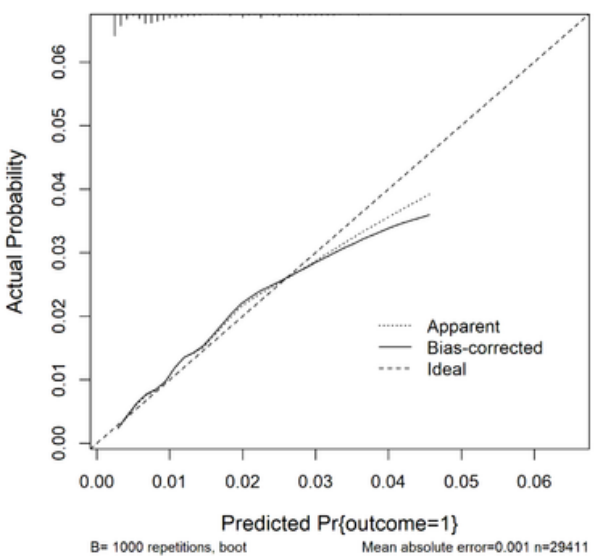

$\mathrm{F}$

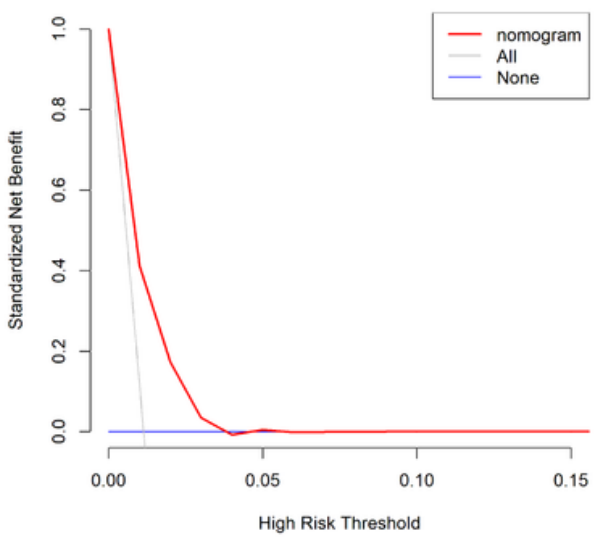

\section{Figure 3}

A-C: Calibration curves showing the probability of the normal control vs esophagitis/LGIN/HGIN and ESCC three groups between the nomogram prediction and the actual observation. Perfect prediction would correspond to a slope of 1 (diagonal 45-degree gray line). D-F: Decision curves of the nomogram predicting the risk. The x-axis represents the threshold probabilities, and the $y$-axis measures the net benefit calculated by adding the true positives and subtracting the false positives. Note: LGIN: low-grade intraepithelial neoplasia; HGIN: high-grade intraepithelial neoplasia; ESCC: esophageal squamous cell carcinoma.

\section{Supplementary Files}

This is a list of supplementary files associated with this preprint. Click to download.

- Supplementaryfile.docx 